\title{
Wearable Sensors in Huntington Disease: A Pilot Study
}

Kelly L. Andrzejewski, DO, PhD, ${ }^{a}$ Ariel V. Dowling, PhD, ${ }^{b}$ David Stamler, MD, ${ }^{\mathrm{c}}$ Timothy J. Felong, BS, ${ }^{\mathrm{d}}$ Denzil A. Harris, BA, Cynthia Wong, BS, ${ }^{\mathrm{c}}$ Hang Cai, MS, ${ }^{\mathrm{b}}$ Ralf Reilmann, MD, PhD, ${ }^{\mathrm{f}}$ Max A. Little, PhD, ${ }^{\text {g, h }}$ Joseph T. Gwin, PhD, ${ }^{\mathrm{b}}$ Kevin M. Biglan, $M D, M P H,{ }^{a, d}$ E. Ray Dorsey, MD, MBA ${ }^{a, d}$

${ }^{a}$ Department of Neurology, University of Rochester Medical Center, Rochester, NY; ${ }^{b}$ BioSensics LLC, Cambridge, MA

${ }^{c}$ Teva Pharmaceuticals, La Jolla, CA; ${ }^{d}$ CHET, University of Rochester Medical Center, Rochester, NY; ${ }^{e}$ University of Rochester School of Medicine and Dentistry, Rochester, NY; ${ }^{f}$ Department of Neurodegenerative Diseases and Hertie-Institute for Clinical Brain Research, University of Tuebingen, Tuebingen, Germany; ${ }^{g}$ Aston University, Birmingham, UK

${ }^{h}$ Massachusetts Institute of Technology, Cambridge, MA

\section{ABSTRACT}

Background: The Unified Huntington's Disease Rating Scale (UHDRS) is the principal means of assessing motor impairment in Huntington disease but is subjective and generally limited to in-clinic assessments.

Objective: To evaluate the feasibility and ability of wearable sensors to measure motor impairment in individuals with Huntington disease in the clinic and at home.

Methods: Participants with Huntington disease and controls were asked to wear five accelerometer-based sensors attached to the chest and each limb for standardized, in-clinic assessments and for one day at home. A second chest sensor was worn for six additional days at home. Gait measures were compared between controls, participants with Huntington disease, and participants with Huntington disease grouped by UHDRS total motor score using Cohen's $d$ values.

Results: Fifteen individuals with Huntington disease and five controls completed the study. Sensor data were successfully captured from 18 of the 20 participants at home. In the clinic, the standard deviation of step time (time between consecutive steps) was increased in Huntington disease ( $p<0.0001$; Cohen's $d=2.61$ ) compared to controls. At home with additional observations, significant differences were observed in seven additional gait measures. The gait of individuals with higher total motor scores ( 50 or more) differed significantly from those with lower total motor scores (below 50) on multiple measures at home.

Conclusions: In this pilot study, the use of wearable sensors in clinic and at home was feasible and demonstrated gait differences between controls, participants with Huntington disease, and participants with Huntington disease grouped by motor impairment.

Keywords: Huntington disease, remote sensing technology, clinical study, movement, gait, chorea, ambulatory monitoring

\section{INTRODUCTION}

The principal means of measuring motor impairment in Huntington disease (HD) is the Unified Huntington's Disease Rating Scale (UHDRS) total motor score [1]. Although valuable, the scale is subjective and categorical $[2,3]$, requires significant training to administer correctly, and only captures impairment in clinic. Quantitative motor tests, notably "Q-Motor" assessments, have helped reduce subjectivity and improve sensitivity of motor assessments in HD [411]. Q-Motor assessments have demonstrated utility in tracking longitudinal progression of motor impairment $[12,13]$ and as a supplemental measure in clinical trials [14]. Likewise, force-sensitive insoles [15,16], video motion analysis systems [17], and pressure-sensitive walking mats [18-20] have been utilized to objectively measure impaired gait in Huntington disease. However, these gait-measuring technologies and Q-Motor assessments must be administered in clinic by trained personnel.

If effective, wearable sensors could enable objective, sensitive, continuous assessment of individuals both in clinic and remotely from home. Previous studies have demonstrated the feasibility and promise of wearable sensors as objective measures of motor impairment in Parkinson disease [21-24]. However, few, if any, published studies have examined such an application in HD. We therefore conducted a pilot study of wearable sensors in clinic and at home in individuals with HD and controls to determine their feasibility and potential utility. 


\section{MATERIALS AND METHODS}

\section{Study overview and design}

We conducted a seven- day, observational study using accelerometer-based, wearable PAMSys- $\mathrm{X}^{\mathrm{TM}}$ sensors $[25,26]$ (BioSensics, Cambridge, MA; $\$ 2,300$ per sensor set) in individuals with HD and controls. Study goals included (1) determining the feasibility of capturing motor assessments with sensors at home, (2) comparing sensor data between participants with HD and controls, and (3) comparing sensor data by motor impairment as measured by the UHDRS total motor score. Participants gave informed, written consent for this study and all procedures were approved by the University of Rochester's institutional review board.

Participants with HD had a clinical diagnosis, either a first degree relative with HD or confirmatory genetic testing (CAG expansion $\geq 36$ ), and a UHDRS total maximal chorea score $\geq 4$ at screening. Unaffected family and friends of participants with HD were recruited as controls. Unaffected family members at risk for HD had previously undergone genetic testing confirming absence of CAG expansion. In clinic, participants provided basic demographic data, completed a survey on technology use, and underwent the Montreal Cognitive Assessment [27]. Participants were then fitted with five sensors affixed by adjustable bands to their ankles, wrists, and chest (Figure 1) and were video recorded while performing the UHDRS motor, Timed Up and Go [28], and Q-Motor assessments. At home, all five sensors were worn for 24 hours (i.e., day 1 ) and then a replacement chest sensor was worn for an additional six days (i.e., days 2-7).

Participants were instructed to remove the non-waterproof sensors when bathing and were given the option to remove them at night, for comfort, while sleeping. Participants mailed the sensors back to BioSensics for data analysis and completed a survey on their experience wearing and perceived utility of the sensors. HD participants with significant cognitive impairment were assisted by family members to ensure all study related activities were completed.

\section{Analysis}

Sensor data analyzed: Tri-axial acceleration data were collected from sensors at a sampling rate of $50 \mathrm{~Hz}$. Only the chest sensor was required for data analysis presented in this paper; wrist and ankle sensor analysis is ongoing and is not included in this report.

Physical activity: Using days 2-7 chest sensor data, active (i.e., non-passive) physical activity measures, including time spent sitting, standing, walking, and running, the total number and duration of sit-to-stand and stand-to-sit transitions, and the total number of walking episodes, steps, and steps per walking episode were computed using commercially available software (PAMWare ${ }^{\mathrm{TM}}$, BioSensics, Cambridge, MA). The software's physical activity analysis algorithms have been described previously [25]. These algorithms use pattern recognition to detect sit-to-stand and stand-to-sit transitions from tri-axial chest acceleration data and a filtering and peak finding scheme to identify episodes of walking from the vertical axis acceleration data.

Gait: Using chest sensor data from days 1 through 7, algorithms were developed to isolate equally weighted walking windows from which gait measures were computed. Using the peak finding scheme from the physical activity analysis software [25], walking episodes defined by 10 or more steps were identified. Within these walking episodes, gait measure values were computed from 15 -second windows of data with $50 \%$ overlap. This overlap was used to ensure that gait cycle events at the beginning or end of a window were not missed. In-clinic gait measures were derived from one walking window during the Timed Up and Go test. Velocity and displacement were calculated from acceleration data by trapezoidal numerical integration and double integration, respectively. Samples of the following gait measures were computed for each isolated 15-second walking window:

Step time standard deviation: Standard deviation of the time between consecutive steps

Cadence: Number of steps per unit time

Step peak acceleration: Maximum and average of the peak acceleration value for each step, which is representative of an individual's heel strike force during footfall

Medial-lateral speed: Maximum and average speed in the side-to-side plane

Medial-lateral displacement: Maximum and average distance in the side-to-side plane

\section{Grouping data and statistics}

For comparing HD versus control, two-tailed, equal variance, unpaired t-tests $(\alpha=0.05)$ were used. For comparing total motor score $\geq 50$, total motor score $<50$, and control, one-way analyses of variance were performed followed by (if 
appropriate) Tukey-Kramer multiple comparison tests $(\alpha=0.05)$. A total motor score of 50 was chosen based mainly on a bimodal distribution. Effect sizes were computed for comparisons using Cohen's $d$ (absolute values).

\section{RESULTS}

\section{Study population and feasibility}

Fifteen participants with HD and five controls completed the one-week study. On average, participants with HD were 56.8 ( \pm 6.6 ) years old, had manifest HD for $7.7( \pm 4.9)$ years, and had a total motor score of $42.3( \pm 13.3)$ and a total maximal chorea score of $9.1( \pm 3.5)$. On average, controls were $53.4( \pm 20.4)$ years old (Table 1$)$. Data were successfully obtained from all participants except for days 2-7 chest sensor recordings for two participants with HD. One sensor had no recorded data, and one sensor had corrupted data due to damage to the memory card. One participant with HD lost an ankle sensor.

\section{Physical activity analysis}

At home, the time spent sitting upright, standing, walking, and running were similar between controls and participants with HD (Figure 2). The total number and duration of sit-to-stand and stand-to-sit transitions and the total number of walking episodes, steps, and steps per walking episode were also similar between controls and participants with HD (data not shown).

\section{Gait analysis: control vs. HD}

In clinic, each participant had one 15-second walking window analyzed. At home (with longer periods of observation), the number of 15 -second walking windows was 14,017 for controls and 36,088 for participants with HD. In clinic, step time standard deviation was increased in HD ( $p<0.0001$; Cohen's $d=2.61)$ (Figure 3A). At home, differences were observed between controls and participants with HD for all eight gait measures $(p<0.0001)$ (Figures $3 A-H)$. Notably, gait cadence was reduced in HD (104.4 \pm 12.1 steps per minute) compared to controls (118.8 \pm 15.5 steps per minute) (Cohen's $d=1.10$ ) (Figure 3B).

\section{Gait analysis: control vs. HD grouped by total motor score}

Of participants with HD, five had a total motor score of at least 50 and 10 had a total motor score of less than 50. However, data from days 2-7 for two participants with total motor scores less than 50 were not successfully captured.

In clinic, step time standard deviation was increased in both sub-groups of participants with HD relative to controls ( $p<0.001$; Cohen's $d \geq 2.94$ ) (Figure 4A). Average step peak acceleration was also increased, in clinic, in those with a total motor score $\geq 50$ relative to controls ( $p<0.05$; Cohen's $d=3.03$ ) (Figure 4D). At home, as the number of walking windows analyzed per group increased, all eight gait measures differed between all three groups $(p<0.01)$ and differed between those with higher and lower total motor scores (Figures 4A-H).

\section{Survey}

The post-study survey was completed by all 20 participants. The majority found the sensors to be "comfortable" $(n=16)$ and "easy to use" $(n=17)$. The most common complaint was that the chest sensor's strap was uncomfortable. When asked if they would use these sensors if asked in the future, 11 of the 15 participants with HD said they would be "very willing."

\section{DISCUSSION}

In this pilot study, the use of wearable sensors at home was feasible, well tolerated, and demonstrated differences in gait among controls, participants with HD, and participants with HD grouped by motor impairment. With more observations (and thus greater power) at home, additional differences in gait were detected. In the uncontrolled setting of the home, the variability in the motor measures detected by the sensors was generally greater than those observed in the controlled clinical environment. However, the vastly greater frequency of observations and thus greater power enabled detection of significant differences.

The results from this study are generally consistent with previous gait assessments conducted in clinic. For example, in-clinic measures have previously shown increased step time variability in HD that increases with disease severity $[15,19]$ and that can differentiate individuals with pre-manifest HD from controls [19]. In this study, in-clinic and at-home results revealed step time standard deviation to be the most sensitive gait measure. Step time standard 
deviation was the sole gait measure found to be different between controls and participants with HD both in clinic and at home. Moreover, relative to other gait measures, step time standard deviation showed the largest effect size when comparing at-home results among individuals with HD grouped by total motor score.

Previous studies have also shown that gait cadence is decreased in HD through in-clinic evaluations [16-19,29]. This study demonstrates that gait cadence is also decreased when measured at home. In addition, the values of at-home steps per minute for controls and participants with HD are very similar to values measured in clinic [16-19].

Medial-lateral velocity and displacement were found to be increased in HD at home. Among these side-to-side movement measures during walking, average medial-lateral velocity had the largest effect size when comparing controls to participants with HD. Previously, using a lower back-attached angular velocity transducer, Grimbergen et al. [20] found medial-lateral trunk sway during walking to be increased in HD and increased in a group of HD "fallers" (with a higher total motor score) relative to "non-fallers." Based on clinical correlation, they attributed trunk sway during walking to chorea.

This pilot study's limitations include its small sample size and short duration. In addition, the magnitude of differences observed in gait, especially at home, was in some cases small, especially among the two sub-groups of individuals with HD. In the future, larger, longer duration studies of wearable sensors will need to further evaluate feasibility, test re-test reliability, and correlate results with standard clinical, and quantitative motor assessments. Larger studies could also include those with prodromal HD and even more advanced HD. While small, this study did suggest that those with modest cognitive impairment could wear sensors.

In addition, future studies could evaluate the ability of sensors to detect changes in response to medications known to improve motor function [30], which could support their greater use in clinical research and potentially patient care. High frequency, objective data acquired in a "real world" setting could also be used to evaluate promising therapeutics in early stage clinical trials and offer the potential to reduce sample size, time, and cost of such trials [3]. Much work remains before evaluating and realizing this promise, but hopefully this study begins to lay the foundation for future evaluations of wearable sensors for assessing important external manifestations of HD.

\section{References}

1. The Huntington Study Group. Unified Huntington's disease rating scale: reliability and consistency. Mov Disord. 1996;11(2):136-42.

2. Reilmann R, Bohlen S, Kirsten F, Ringelstein EB, Lange HW. Assessment of involuntary choreatic movements in Huntington's disease: toward objective and quantitative measures. Mov Disord. 2011;26(12):2267-73.

3. Dorsey ER, Venuto C, Venkataraman V, Harris DA, Kieburtz K. Novel methods and technologies for $21^{\text {st }}$-century clinical trials. JAMA Neurol. 2015;72(5):582-8.

4. Gordon AM, Quinn L, Reilmann R, Marder K. Coordination of prehensile forces during precision grip in Huntington's disease. Exp Neurol. 2000;163(1):136-48.

5. Quinn L, Reilmann R, Marder K, Gordon AM. Altered movement trajectories and force control during object transport in Huntington's disease. Mov Disord. 2001;16(3):469-80.

6. Reilmann R, Kirsten F, Quinn L, Henningsen H, Marder K, Gordon AM. Objective assessment of progression in Huntington's disease: a 3-year follow-up study. Neurology. 2001;57(5):920-4.

7. Tabrizi SJ, Langbehn DR, Leavitt BR, et al. Biological and clinical manifestations of Huntington's disease in the longitudinal TRACK-HD study: cross-sectional analysis of baseline data. Lancet Neurol. 2009;8(9):791-801.

8. Reilmann R, Bohlen S, Klopstock T, et al. Grasping premanifest Huntington's disease - shaping new endpoints for new trials. Mov Disord. 2010;25(16):2858-62.

9. Bechtel N, Scahill RI, Rosas HD, et al. Tapping linked to function and structure in premanifest and symptomatic Huntington disease. Neurology. 2010;75(24):2150-60.

10. Reilmann R, Bohlen S, Klopstock T, et al. Tongue force analysis assesses motor phenotype in premanifest and symptomatic Huntington's disease. Mov Disord. 2010;25(13):2195-202.

11. Scahill RI, Hobbs NZ, Say MJ, et al. Clinical impairment in premanifest and early Huntington's disease is associated with regionally specific atrophy. Hum Brain Mapp. 2013(3);34:519-29.

12. Tabrizi SJ, Scahill RI, Durr A, et al. Biological and clinical changes in premanifest and early stage Huntington's disease in the TRACK-HD study: the 12-month longitudinal analysis. Lancet Neurol. 2011;10(1):31-42.

13. Tabrizi SJ, Scahill RI, Owen G, et al. Predictors of phenotypic progression and disease onset in premanifest and early-stage Huntington's disease in the TRACK-HD study: analysis of 36-month observational data. Lancet Neurol. 2013;12(7):637-49. 
14. Reilmann R, Rouzade-Dominguez ML, Saft C, et al. A randomized, placebo-controlled trial of AFQ056 for the treatment of chorea in Huntington's disease. Mov Disord. 2015;30(3):427-31.

15. Hausdorff JM, Cudkowicz ME, Firtion R, Wei JY, Goldberger AL. Gait variability and basal ganglia disorders: stride-to-stride variations of gait cycle timing in Parkinson's disease and Huntington's disease. Mov Disord. 1998;13(3):428-37.

16. Bilney B, Morris ME, Churchyard A, Chiu E, Georgiou-Karistianis N. Evidence for a disorder of locomotor timing in Huntington's disease. Mov Disord. 2005;20(1):51-7.

17. Delval A, Krystkowiak P, Blatt JL, Labyt E, Dujardin K, Destee A, et al. Role of hypokinesia and bradykinesia in gait disturbances in Huntington's disease: a biomechanical study. J Neurol. 2006;253(1):73-80.

18. Rao AK, Quinn L, Marder KS. Reliability of spatiotemporal gait outcome measures in Huntington's disease. Mov Disord. 2005;20(8):1033-7.

19. Rao AK, Muratori L, Louis ED, Moskowitz CB, Marder KS. Spectrum of gait impairments in presymptomatic and symptomatic Huntington's disease. Mov Disord. 2008;23(8):1100-7.

20. Grimbergen YAM, Knol MJ, Bloem BR, Kremer BPH, Roos RAC, Munneke M. Falls and gait disturbances in Huntington's disease. Mov Disord. 2008;23(7):970-6.

21. Patel S, Lorincz K, Hughes R, et al. Monitoring motor fluctuations in patients with Parkinson's disease using wearable sensors. IEEE Trans Inf Technol Biomed. 2009;13(6):864-73.

22. Chen BR, Patel S, Buckley T, et al. A web-based system for home monitoring of patients with Parkinson's disease using wearable sensors. IEEE Trans Biomed Eng. 2011;58(3):831-6.

23. Patel S, Chen BR, Mancinelli C, et al. Longitudinal monitoring of patients with Parkinson's disease via wearable sensor technology in the home setting. Conf Proc IEEE Eng Med Biol Soc. 2011;1552-5.

24. Toosizadeh N, Mohler J, Lei H, Parvaneh S, Sherman S, Najafi B. Motor performance assessment in Parkinson's disease: association between objective in-clinic, objective in-home, and subjective/semi-objective measures. PLoS One. 2015;10(4):e0124763.

25. Najafi B, Aminian K, Paraschiv-lonescu A, Loew F, Bula CJ, Robert P. Ambulatory system for human motion analysis using a kinematic sensor: monitoring of daily physical activity in the elderly. IEEE Trans Biomed Eng. 2003;50(6):711-23.

26. Najafi B, Armstrong DG, Mohler J. Novel wearable technology for assessing spontaneous daily physical activity and risk of falling in older adults with diabetes. J Diabetes Sci Technol. 2013;7(5):1147-60.

27. Nasreddine ZS, Phillips NA, Bedirian V, et al. The Montreal Cognitive Assessment, MoCA: a brief screening tool for mild cognitive impairment. J Am Geriatr Soc. 2005;53(4):695-9.

28. Podsiadlo D, Richardson S. The timed "Up \& Go": a test of basic functional mobility for frail elderly persons. J Am Geriatr Soc. 1991;39(2):142-8.

29. Koller WC, Trimble J. The gait abnormality of Huntington's disease. Neurology. 1985;35(10):1450-4.

30. Frank SA. First-time use of SD-809 in Huntington disease (first-HD). Neurology. 2015;85(4):e47. 
Table 1. Baseline characteristics of the study population

\begin{tabular}{|c|c|c|}
\hline & $\begin{array}{l}\mathrm{HD} \\
(\mathrm{n}=15)\end{array}$ & $\begin{array}{l}\text { Controls } \\
(n=5)\end{array}$ \\
\hline \multicolumn{3}{|l|}{ Demographics } \\
\hline Age (years) & $56.8 \pm 6.6$ & $53.4 \pm 20.4$ \\
\hline Women [\%] & 40 & 60 \\
\hline White [\%] & 100 & 100 \\
\hline Hispanic ethnicity [\%] & 0 & 0 \\
\hline 4-year college degree or higher education [\%] & 40 & 60 \\
\hline Currently employed or a student [\%] & 0 & 60 \\
\hline Currently married or in a domestic partnership [\%] & 60 & 20 \\
\hline \multicolumn{3}{|l|}{ HD Characteristics } \\
\hline Years since diagnosis & $7.7 \pm 4.9$ & \\
\hline Currently seeing an HD specialist [\%] & 100 & \\
\hline UHDRS total motor score (0-124) & $42.3 \pm 13.3$ & \\
\hline UHDRS total maximal chorea score $(0-28)$ & $9.1 \pm 3.5$ & \\
\hline Timed Up and Go (seconds) & $16.2 \pm 5.2$ & $11.7 \pm 2.8$ \\
\hline 10 Meter Walk Test (seconds) & $12.2 \pm 5.5$ & $7.5 \pm 1.1$ \\
\hline Montreal Cognitive Assessment score (0-30) & $21.5 \pm 3.7$ & $28.2 \pm 0.8$ \\
\hline \multicolumn{3}{|l|}{ Internet and Technology Use } \\
\hline Use Internet or email [\%] & 80 & 80 \\
\hline Have used Internet to look up health information [\%] & 73 & 80 \\
\hline $\begin{array}{l}\text { Have used smartphone or tablet to access Internet } \\
\text { [\%] }\end{array}$ & 27 & 60 \\
\hline $\begin{array}{l}\text { Have used computer or other device to video chat } \\
\text { [\%] }\end{array}$ & 27 & 60 \\
\hline
\end{tabular}

Values are mean \pm standard deviation unless noted as [\%] UHDRS, Unified Huntington's Disease Rating Scale 


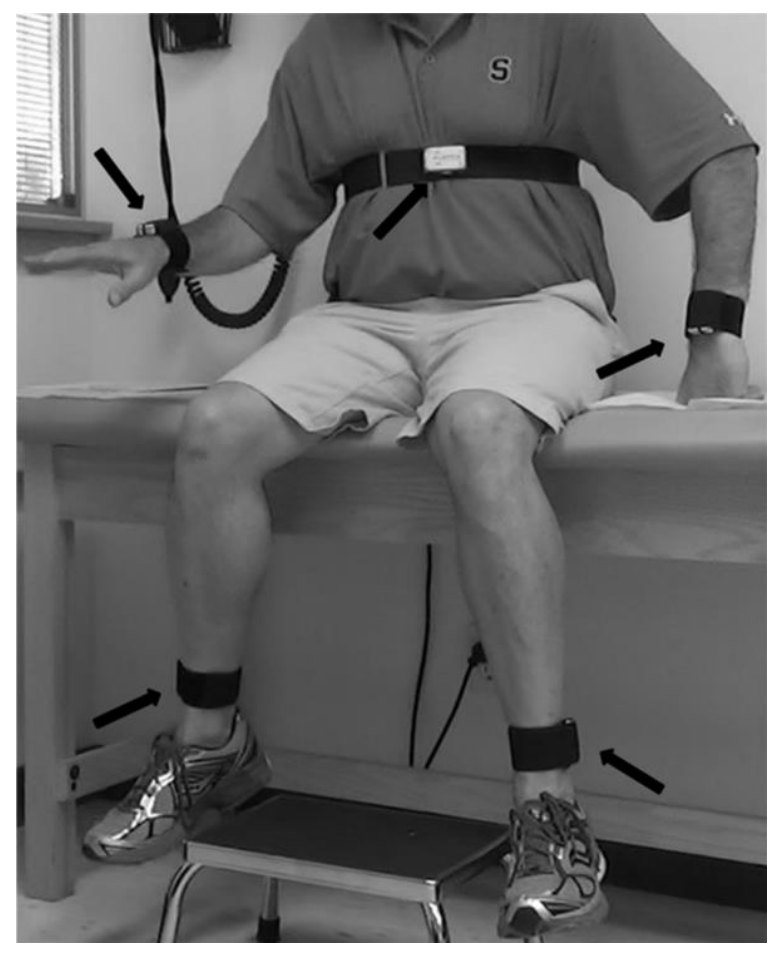

Figure 1. Picture of wearable chest, ankle, and wrist sensors used in the study.

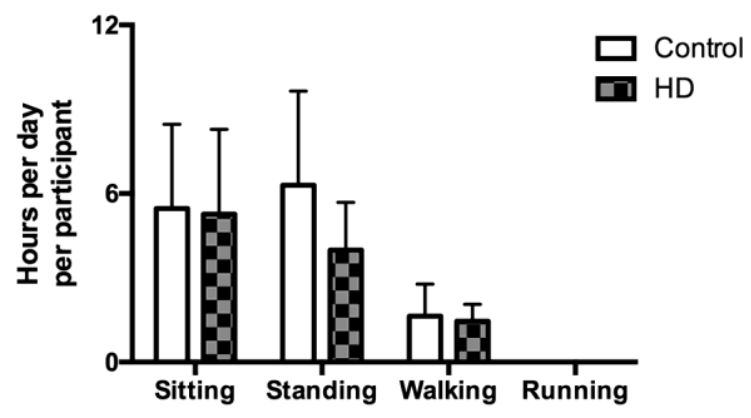

Figure 2. Active (i.e., non-passive) physical activity measures comparing controls and participants with HD. 


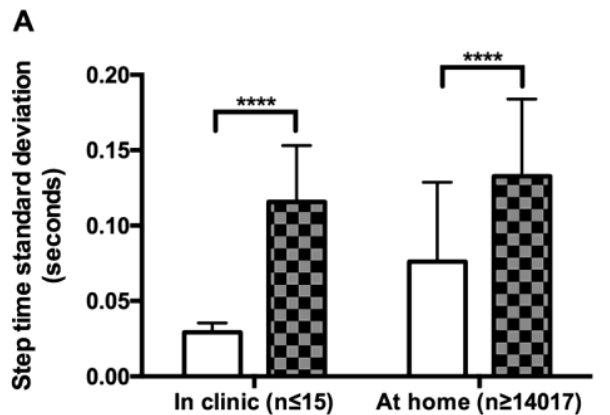

C

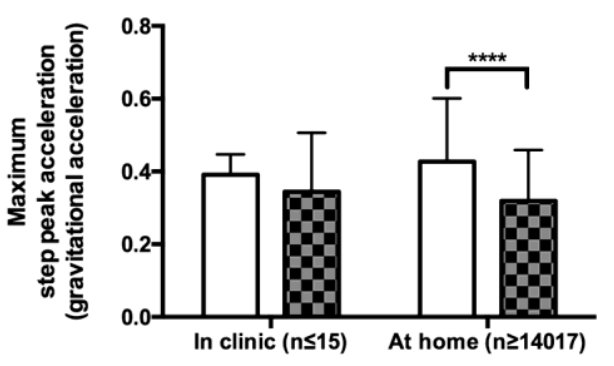

E

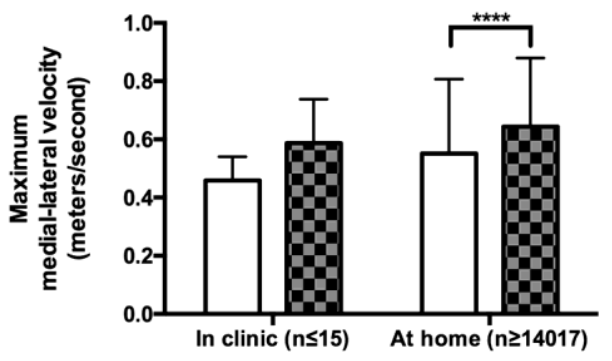

G

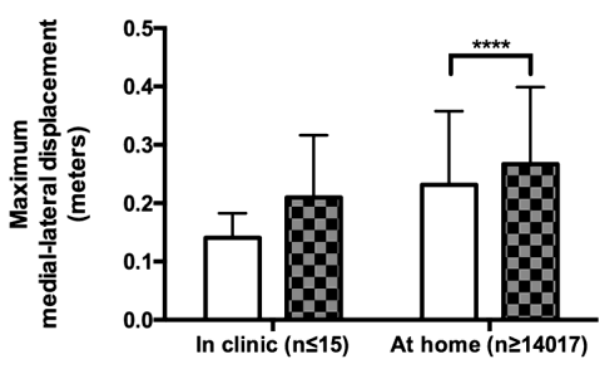

B
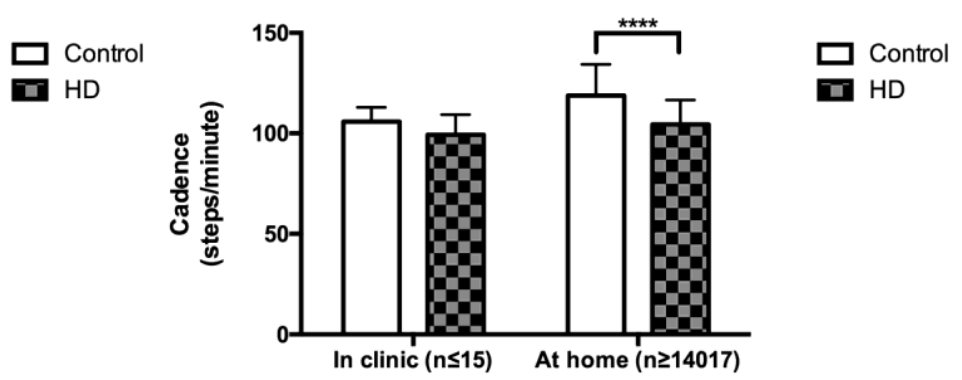

D

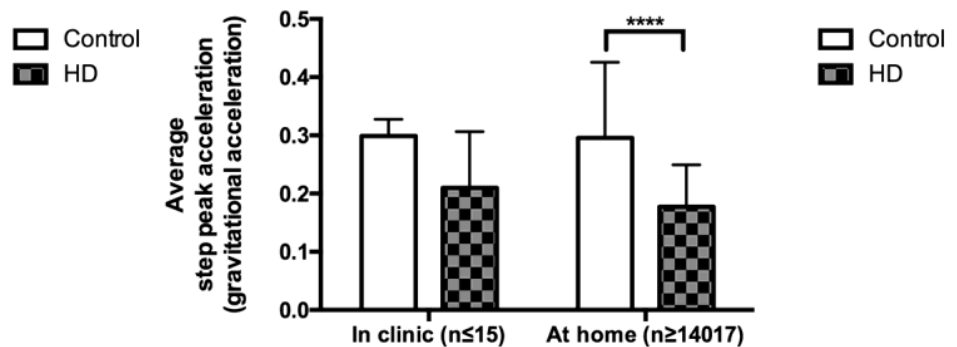

$\mathbf{F}$

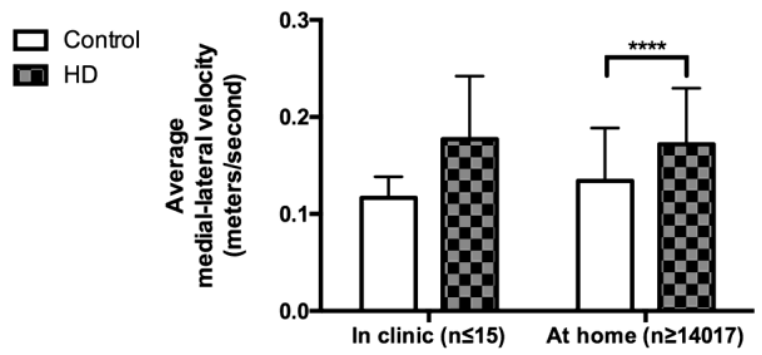

H
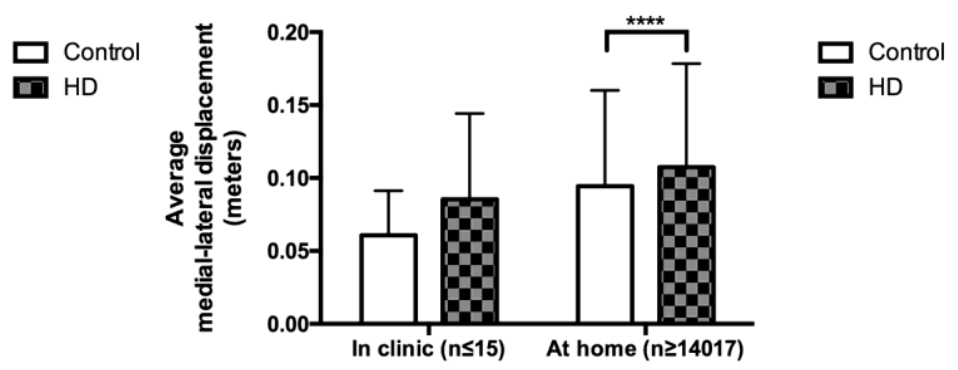

Figure 3. In-clinic and at-home gait measures comparing controls and participants with $\mathrm{HD}\left({ }^{*} p<0.05\right.$; $\left.{ }^{* *} p<0.01 ;{ }^{* * *} p<0.001 ; * * * * p<0.0001\right)$. 
A

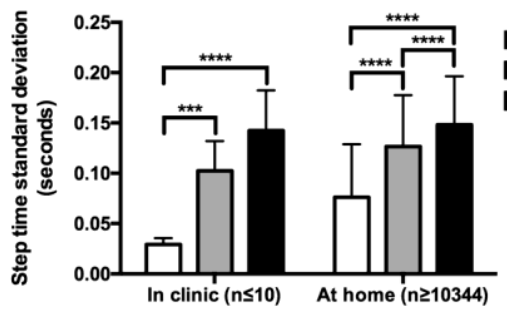

C

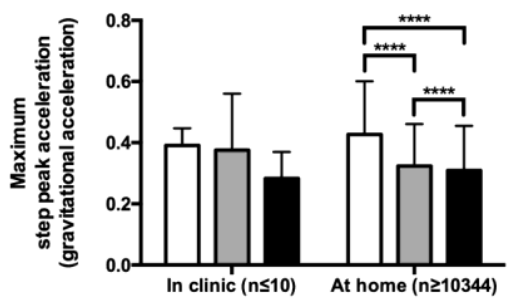

E

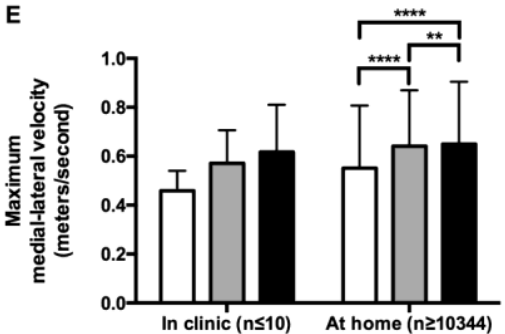

G

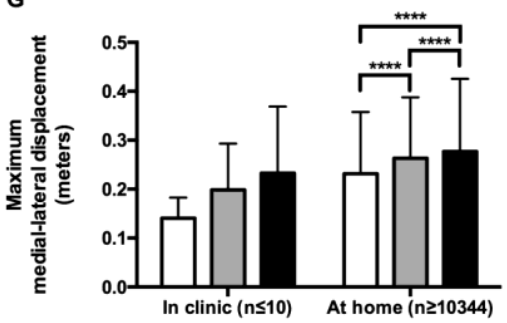

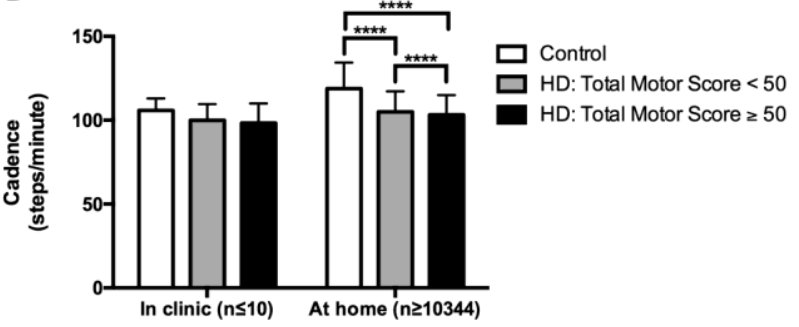

D

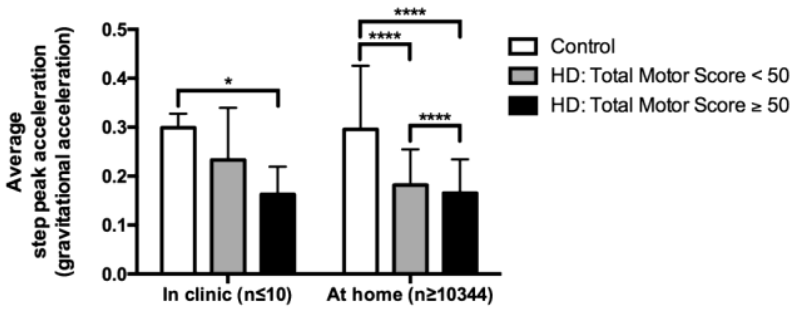

$\mathbf{F}$

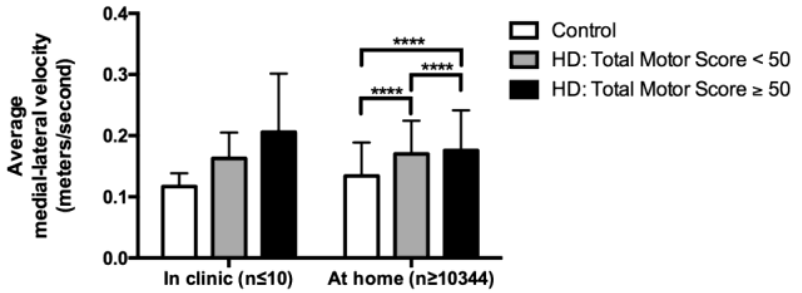

H

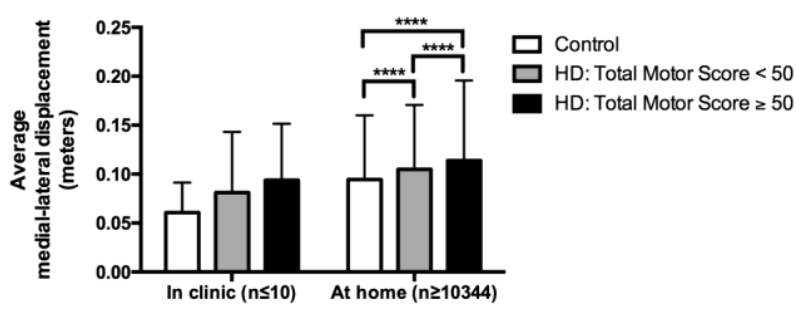

Figure 4. In-clinic and at-home gait measures comparing controls and participants with HD grouped by total motor score $\left({ }^{*} p<0.05 ;{ }^{* *} p<0.01 ;{ }^{* *} p<0.001 ;{ }^{* * * *} p<0.0001\right)$. 